\title{
Erratum: Transport equation approach to calculations of Hadamard Green functions and noncoincident DeWitt coefficients [Phys. Rev. D 84, 104039 (2011)]
}

Adrian C. Ottewill and Barry Wardellø

(Q) (Received 10 January 2020; published 24 January 2020)

DOI: 10.1103/PhysRevD.101.029901

There was a nonpropagating sign error in Eq. (3.24). The corrected equation is

$$
D^{\prime} A_{a b c}+A_{a b \alpha} \xi^{\beta^{\prime}}{ }_{\gamma^{\prime}} g_{\beta^{\prime}}{ }^{\alpha} g_{c}{ }^{\gamma^{\prime}}-g_{a}{ }^{\alpha^{\prime}} g_{b}{ }^{\beta^{\prime}} g_{c}{ }^{\gamma^{\prime}} R_{\alpha^{\prime} \beta^{\prime} \gamma^{\prime} \delta^{\prime}} \sigma^{\delta^{\prime}}=0 .
$$

We thank Justin Vines, Jia Shouqing, and Eoin Murphy for independently identifying this sign error. 